\title{
Lixiviação de Herbicidas no Solo Aplicados com Água de IRRIGAÇÃo EM Plantio Direto ${ }^{1}$
}

\author{
Leaching of Herbicides Applied with Irrigation Water Under No-till \\ Systems
}

FONTES, J.R.A. ${ }^{2}$, SILVA, A.A. ${ }^{3}$, VIEIRA, R.F.. ${ }^{4}$ e RAMOS, M.M. ${ }^{5}$

\begin{abstract}
RESUMO - O objetivo deste trabalho foi avaliar a lixiviação no solo dos herbicidas metolachlor e fomesafen aplicados com água de irrigação de pivô central, nos plantios direto (palha de milho e de plantas daninhas) e convencional, na cultura do feijão (Phaseolus vulgaris) em um Argissolo Vermelho-Amarelo câmbico. Foi conduzido um experimento, com três repetições, no qual o metolachlor $\left(2,4 \mathrm{~kg} \mathrm{ha}^{-1}\right)$ foi aplicado, em pré-emergência, com lâminas de água de 5, 10 e $15 \mathrm{~mm}$, e o fomesafen $\left(0,225 \mathrm{~kg} \mathrm{ha}^{-1}\right)$, em pós-emergência, com 3, 6 e $9 \mathrm{~mm}$. Os herbicidas também foram aplicados com pulverizador costal. Para a avaliação da lixiviação, foram conduzidos bioensaios em casa de vegetação, coletando-se amostras de solo, quinzenalmente, nas camadas de 0-5, 5-10 e 10-15 cm de profundidade, num total de três repetições. A primeira amostra, de um total de quatro, foi retirada 15 dias após a aplicação. Como planta-teste foi utilizado sorgo (Sorghum bicolor), híbrido BR 304. Quinze dias após a semeadura, as plantas foram coletadas inteiras (parte aérea e raízes) para a determinação da massa seca. No plantio direto, o metolachlor, independentemente do método de aplicação, não sofreu lixiviação nem apresentou atividade no solo aos 15 dias após a aplicação. No plantio convencional, a herbigação do metolachlor só afetou o crescimento do sorgo cultivado na camada de solo mais superficial $(0-5 \mathrm{~cm}$ de profundidade) aos 15 dias após a aplicação. O fomesafen, independentemente do método de aplicação e do sistema de plantio, foi lixiviado até $10 \mathrm{~cm}$ de profundidade e detectado até na última data de amostragem (60 dias após a aplicação). A aplicação do metolachlor e do fomesafen com água de irrigação apresentou-se segura em relação à movimentação no solo.
\end{abstract}

Palavras-chave: herbigação, metolachlor, fomesafen, sistemas de plantio.

ABSTRACT - The aim of this study was to evaluate the leaching of the herbicides metolachlor and fomesafen in an Alfisol soil trough irrigation water under no-till and conventional tillage systems. One experiment consisted of three replications with metolachlor $\left(2,4 \mathrm{~kg} \mathrm{ha}^{-1}\right)$ applied in pre-emergence, at water depths of 5, 10 and $15 \mathrm{~mm}$, and fomesafen $\left(0,225 \mathrm{~kg} \mathrm{ha}^{-1}\right)$ applied in post-emergence, at water depths of 3, 6 and $9 \mathrm{~mm}$. The herbicides had also been applied by spraying. Bioassays were carried out under greenhouse conditions using soil samples from the plots. The soil samples were taken each fifteen days from layers of 0-5, 5-10 and 10-15 cm depth. The first sample, out of a total of four, was collected 15 days after herbicides aplication. The samples were sowed with sorghum bicolor, hybrid BR 304, which was used as test-plant. Fifteen days after sowing, the whole plants (shoot and roots) were collected for dry mass weight. Under no-till, metolachlor was not detected in the soil at 15 days after aplication, regardless of the application method. Under the conventional system, only metolachlor herbigation affected sorghum growth at the $0-5 \mathrm{~cm}$ soil layer. Fomesafen was leached down to $10 \mathrm{~cm}$ depth, being detected at the last sampling date (60 days after application), regardless of the application methods. Herbigation of metolachlor and fomesafen showed to be reliable in relation to soil movement.

Key words: herbigation, metolachlor, fomesafen, tillage systems.

1 Recebido para publicação em 4.2.1002 e na forma revisada em 17.12.2004.

2 Eng.-Agr., D.S., Técnico de Nível Superior, Embrapa Cerrados, Caixa Postal 08223, 73310-970 Planaltina-DF, $<$ roberto@cpac.embrapa.br>. ${ }^{3}$ Eng.-Agr., D.S., Professor do Departamento de Fitotecnia da Universidade Federal de Viçosa UFV, 36570-000 Viçosa-MG; ${ }^{4}$ Eng.-Agr., D.S., Pesquisador da Embrapa-Epamig, Viçosa-MG, ${ }^{5}$ Eng.-Agr., D.S., Professor do Departamento de Engenharia Agrícola - UFV. 


\section{INTRODUÇÃO}

A aplicação de herbicidas para o controle de plantas daninhas é prática comum na cultura do feijão, e os produtores que utilizam irrigação têm aplicado esses produtos com a água (herbigação), normalmente com pivô central, principalmente em virtude da redução do custo de aplicação e mão-de-obra (Vieira \& Silva, 1998). Entretanto, são poucos os trabalhos de pesquisa que avaliaram os efeitos da herbigação sobre a eficácia de controle de plantas daninhas e o comportamento de herbicidas no ambiente. Questão relevante é a movimentação de herbicidas no solo, por causa do perigo de contaminação de águas subterrâneas e de corpos de água.

A lixiviação é o carreamento do herbicida em solução para baixo, no solo, por meio da força gravitacional (Keller \& Weber, 1997), das características físico-químicas dos herbicidas (do gradiente de pressão de vapor e difusão), do tipo de solo e das condições climáticas (Lee \& Weber, 1993; Oliveira Jr., 1998). A mobilidade e persistência de herbicida no solo são os principais indicadores de seu potencial para contaminação de corpos de água e do lençol freático (Weber et al., 1993; Gustafson, 1989; Inoue et al., 2003).

Quanto aos efeitos de sistema de plantio sobre a lixiviação de herbicidas, os resultados dos trabalhos não são consistentes. Starr \& Glotfelty (1990) e Ritter et al. (1994) avaliaram a movimentação do atrazine em solos francosiltoso e franco-arenoso e não verificaram diferenças na lixiviação do herbicida, comparando o plantio direto com o convencional.

Todavia, de acordo com Dao (1995), o aumento da biomassa de resíduos vegetais sobre o solo pode inibir a lixiviação de herbicidas, devido à sua interceptação e sorção nesses resíduos. Esse autor verificou mais movimentação de atrazine em áreas de plantio convencional que em plantio direto. Estudos têm mostrado que os resíduos vegetais possuem maior capacidade de sorção que o solo (Reddy et al., 1995).

Em alguns casos, mais lixiviação de herbicidas foi verificada nos solos em área de plantio direto (Isensee et al., 1990; Hall et al., 1989; Keller \& Weber, 1997). Sigua et al. (1993) e Isensee \& Sadeghi (1995) atribuem esse fato ao menor escoamento superficial e à maior infiltração de água nesse sistema de plantio. Além disso, no plantio direto é maior o conteúdo de matéria orgânica na superfície do solo, o que aumenta a estabilidade de agregados, a quantidade de macroporos e o maior volume de canais formados por atividade de macrofauna e por raízes decompostas (Heatwole et al., 1997). Por isso, há mais lixiviação de herbicidas, principalmente em condição de saturação de água (Wilson et al., 1998).

O metolachlor apresenta de fraca (Rodrigues \& Almeida, 1998) a moderada (Keller et al., 1998; Vieira \& Silva, 1998) lixiviação no solo, mostrando potencial de contaminação do lençol freático (Gustafson, 1989; Inoue et al., 2003). Sua solubilidade em água, a $20{ }^{\circ} \mathrm{C}$, é de $448 \mu \mathrm{L} \mathrm{L}^{-1}$ e seu coeficiente de partição carbono orgânico-água $\left(\mathrm{K}_{\mathrm{OC}}\right)$ é de $200 \mathrm{~mL} \mathrm{~g}^{-1}$ de solo (Rodrigues \& Almeida, 1998). Trabalhos mostraram que a profundidade de lixiviação do metolachlor depende da ocorrência e intensidade de chuvas ou irrigação e dos teores de argila e de matéria orgânica do solo (Burgard et al., 1993; Procópio et al., 2001). Segundo Keller et al. (1998), a sorção do metolachlor no solo correlaciona-se positivamente com o conteúdo de ácidos húmicos, matéria orgânica e argilas. Wiese e Turner (1977) e Barnes et al. (1992) aplicaram o metolachlor com água de irrigação de um pivô central e avaliaram a sua lixiviação em diferentes solos, constatando não haver influência do método de aplicação sobre sua lixiviação.

O fomesafen apresenta solubilidade em água de $600.000 \mu \mathrm{L} \mathrm{L^{-1 }}$ e $\mathrm{K}_{\mathrm{OC}}$ de $60 \mathrm{~mL} \mathrm{~g}^{-1} \mathrm{de}$ solo (Rodrigues \& Almeida, 1998), também apresentando, a exemplo do metolachlor, potencial de contaminação do lençol freático (Gustafson, 1989). Weber et al. (1993) avaliaram a movimentação do fomesafen em colunas de solo e constataram que $94 \%$ da quantidade aplicada concentrou-se na profundidade de até $17,5 \mathrm{~cm}$, depois da aplicação de lâmina de água de $500 \mathrm{~mm}\left(12,5 \mathrm{~mm} \mathrm{dia}^{-1}\right)$. Cobucci (1996) avaliou a lixiviação em campo do fomesafen em um Latossolo Vermelho-Escuro e verificou que este herbicida $\left(0,125\right.$ a $\left.0,50 \mathrm{~kg} \mathrm{ha}^{-1}\right)$, aplicado por pulverização (pulverizador costal pressurizado com $\mathrm{CO}_{2}$ ), ficou concentrado na 
camada de solo de $0-10 \mathrm{~cm}$ de profundidade. A baixa mobilidade do fomesafen nos solos pode ser atribuída à sua sorção aos colóides orgânicos e minerais (Weber, 1993; Weber et al., 1993) e também aos óxidos de ferro e alumínio (Cobucci, 1996).

O objetivo deste trabalho foi estudar os efeitos de métodos de aplicação de herbicidas (herbigação e pulverização) na cultura do feijão na lixiviação dos herbicidas metolachlor e fomesafen no solo, em plantios direto e convencional.

\section{MATERIAL E MÉTODOS}

Este trabalho foi conduzido na Fazenda Experimental da Universidade Federal de Viçosa, em um Argissolo Vermelho-Amarelo câmbico, cujas características físicas e de matéria orgânica são apresentadas na Tabela 1.

O trabalho constou de um experimento em que foram aplicados o metolachlor $\left(2,4 \mathrm{~kg} \mathrm{ha}^{-1}\right)$, em pré-emergência, e o fomesafen $\left(0,225 \mathrm{~kg} \mathrm{ha}^{-1}\right)$, em pós-emergência. O estudo foi conduzido em área irrigada por pivô central, metade da qual foi cultivada em plantio direto e metade em plantio convencional. A área do plantio direto foi formada sobre palhada de milho e vegetação daninha, dessecada com a aplicação da mistura dos herbicidas glyphosate e 2,4-D $\left(1,08+0,72 \mathrm{~kg} \mathrm{ha}^{-1}\right.$, respectivamente) 15 dias antes da semeadura. No plantio convencional, o preparo de solo foi realizado com uma aração e duas gradagens, um dia antes da semeadura do feijão.

Em ambos os sistemas de plantio, os herbicidas foram aplicados por meio da água de irrigação e por pulverização. Na herbigação, o metolachlor foi aplicado com lâminas de água de 5,10 e $15 \mathrm{~mm}$, e o fomesafen, com 3, 6 e $9 \mathrm{~mm}$.

A área experimental foi dividida em 12 setores com forma de cunha, cada uma com ângulo de $30^{\circ}$. As herbigações foram feitas alternadamente em seis setores úteis, de modo que entre dois setores úteis houvesse um setor onde o pivô central pudesse ser movimentado para iniciar e/ou finalizar a aplicação de determinada lâmina. As parcelas foram locadasentre 70 e $80 \mathrm{~m}$ em relação ao centro do pivô. Os tratamentos de pulverização, por independerem das lâminas de água aplicadas, tiveram apenas uma parcela locada em cada um dos setores úteis.

Na pulverização, os herbicidas foram aplicados com pulverizador pressurizado com $\mathrm{CO}_{2}$, com barra de 2,0 m de comprimento com quatro pontas de pulverização TT 110.02; manteve-se constante a pressão em $3 \mathrm{kgf} \mathrm{cm}^{-2} \mathrm{e}$ aplicou-se volume de calda equivalente a $200 \mathrm{~L} \mathrm{ha}^{-1}$. Foi incluída uma testemunha sem aplicação de herbicidas. Portanto, o experimento constou de 17 tratamentos. O metolachlor foi aplicado um dia após a semeadura do feijão, em ambos os sistemas de plantio, e o fomesafen, 25 dias após a emergência do feijão, quando as plantas daninhas apresentavam de um a dois pares de folhas.

A semeadura da variedade de feijão Pérola foi realizada em 28/7/1999, por meio de semeadora para plantio direto, também ajustada para a área arada e gradeada, posicionando as sementes a $4 \mathrm{~cm}$ de profundidade. O espaçamento entre fileiras foi de $0,45 \mathrm{~m}$, sendo distribuídas 15 sementes por metro de sulco. A adubação de plantio constou de $600 \mathrm{~kg} \mathrm{ha}^{-1} \mathrm{da}$ formulação 4-14-8 (N- $\left.\mathrm{P}_{2} \mathrm{O}_{5}-\mathrm{K}_{2} \mathrm{O}\right)$. A adubação em cobertura foi feita 32 dias após a semeadura, por fertirrigação. Foram distribuídos $45 \mathrm{~kg}$ de $\mathrm{N} \mathrm{ha}^{-1}$ na forma de uréia.

As irrigações da lavoura tiveram início quatro dias após a aplicação do metolachlor. Até a data da primeira amostragem (15 dias após a aplicação) foram aplicados na área $53 \mathrm{~mm}$ de água.

Foi empregado um pivô central de média pressão. Os coeficientes de uniformidade

Tabela 1 - Análise textural e de matéria orgânica de amostras do solo coletadas na área experimental, na camada de 0-15 cm de profundidade. Coimbra-MG, 1999-

\begin{tabular}{|l|c|c|}
\hline \multicolumn{1}{|c|}{ Característica } & Plantio Direto & $\begin{array}{c}\text { Plantio } \\
\text { Convencional }\end{array}$ \\
\hline Areia grossa $\left(\mathrm{dag} \mathrm{kg}^{-1}\right)$ & 26,0 & 33,0 \\
\hline Areia fina $\left(\mathrm{dag} \mathrm{kg}^{-1}\right)$ & 14,0 & 14,0 \\
\hline Silte $\left(\mathrm{dag} \mathrm{kg}^{-1}\right)$ & 11,0 & 10,0 \\
\hline Argila $($ dag kg & -1 & 4,0 \\
\hline Classificação textural & 49,0 & 43,0 \\
\hline Matéria orgânica $($ dag kg & Argila & Argila-arenosa \\
\hline
\end{tabular}

${ }^{1 /}$ Análises realizadas nos Laboratórios de Física do Solo e de Rotina do Departamento de Solos da Universidade Federal de Viçosa. 2/ Método de Walkley e Black. 
de distribuição de água do sistema foram determinados segundo a metodologia da Associação...-ABNT (1985). Durante a herbigação com o metolachlor, os coeficientes de uniformidade de Christiansen (CUC) foram de 91,5 e 91,9\% no plantio direto e no convencional, respectivamente; e durante a herbigação do fomesafen, de 87,1 e 90,1\%, respectivamente. De acordo com Mantovani \& Ramos (1994), esses valores são ótimos para pivô central, indicando boa uniformidade de distribuição de água; segundo Threadgill (1985), eles são adequados para aplicação de defensivos via pivô central. Para a injeção da solução herbicida na água de irrigação utilizou-se uma bomba hidráulica de diafragma.

Durante as herbigações com uma lâmina de água, as parcelas com outras lâminas ou tratamentos foram cobertas com polietileno transparente (colocadas sobre armações de metal). Essas armações tinham 3,0 m de comprimento e 1,8 m de largura. Portanto, a parcela experimental foi constituída por quatro fileiras com 3,0 m de comprimento e 1,8 m de largura, ou seja, do mesmo tamanho das parcelas experimentais, que foram formadas por quatro fileiras de feijão. A área útil constou das duas fileiras centrais, descontando-se $0,5 \mathrm{~m}$ em cada extremidade, isto é, ela foi de $1,8 \mathrm{~m}^{2}$.

A avaliação da lixiviação do metolachor e do fomesafen no solo foi feita por meio de bioensaios. Para isso, amostras de solo foram retiradas, quinzenalmente, nas camadas de 0-5, 5-10 e 10-15 cm de profundidade. A primeira amostragem, num total de quatro, foi feita 15 dias após a aplicação dos herbicidas. As amostras, uma por parcela, foram retiradas com enxadão, pá reta e faca. A fim de evitar contaminação das amostras, utilizou-se éter metílico para se proceder à descontaminação das ferramentas entre amostragens. As amostras foram colocadas em sacos plásticos e levadas no mesmo dia para a casa de vegetação. Em seguida, foram colocadas em vasos plásticos de $300 \mathrm{~cm}^{3}$ de capacidade, sendo, então, efetuada a semeadura do sorgo (Sorghum bicolor), híbrido BR 304, utilizado como planta-teste. Foram usadas 12 sementes por vaso. Após a emergência das plântulas, foi realizado desbaste, deixando oito plantas por vaso. Quinze dias após a semeadura, as plantas foram coletadas inteiras (parte aérea e raízes) e secas em estufa com circulação forçada de ar, a $72^{\circ} \mathrm{C}$, até atingirem peso constante, para determinação da massa seca. Considerou-se como $100 \%$ a produção de massa seca do tratamento testemunha. Os bioensaios foram conduzidos com três repetições. Na interpretação dos resultados utilizou-se a estatística descritiva, com apresentação das médias de tratamentos com seus respectivos erros-padrão. Nas herbigações, as médias foram calculadas com base nos resultados das três repetições dentro de cada lâmina de água e para cada sistema de plantio. No caso da pulverização e da testemunha, utilizaram-se, para os cálculos, os dados obtidos nas três parcelas distribuidas em cada sistema de plantio (uma parcela em cada setor útil).

\section{RESULTADOS E DISCUSSÃO}

Observa-se, na Tabela 2, que, 15 dias após a aplicação, o metolachlor só foi detectado na camada de $0-5 \mathrm{~cm}$ de solo na área do plantio convencional e quando se utilizou a herbigação. Na pulverização, a não-detecção do herbicida na camada de $0-5 \mathrm{~cm}$ foi devido, provavelmente, às maiores perdas do produto por fotodecomposição e/ou volatilização, em relação às herbigações. Aplicado via água de irrigação, mesmo com lâminas de água de $5 \mathrm{~mm}$, o herbicida parece ter sido suficientemente incorporado ao solo para reduzir tais perdas.

Vale ressaltar que a lavoura só começou a ser irrigada quatro dias após a aplicação do metolachlor. Até a data da primeira amostragem foram aplicados na área $53 \mathrm{~mm}$ de água. Segundo Ahrens (1994), as perdas do metolachlor por volatilização são geralmente baixas. A fotodecomposição, no entanto, é a principal causa de dissipação do produto, principalmente em condições secas e quando o metolachlor permanece na superficie do solo.

Nas avaliações feitas aos 30, 45 e 60 dias após a aplicação do metolachlor (Tabelas 3, 4 e 5), a planta-teste geralmente não acusou a presença do herbicida.

Esses resultados confirmam a meia-vida relativamente curta desse herbicida no solo. Segundo Rodrigues \& Almeida (1998), o metolachlor tem persistência média no solo de 
15 a 50 dias, dependendo do tipo de solo, do contéudo de matéria orgânica e das condições edafoclimáticas.

Segundo Ahrens (1994), a lixiviação do metolachlor geralmente é insignificante quando o conteúdo de matéria orgânica do solo é maior que $2 \mathrm{dag}_{\mathrm{kg}}{ }^{-1}$, mesmo não apresentando valor elevado de $\mathrm{K}_{\mathrm{OC}}\left(200 \mathrm{~mL} \mathrm{~g}^{-1}\right.$ de solo) e mostrando solubilidade em água considerada elevada ( $448 \mu \mathrm{L} \mathrm{L} \mathrm{L}^{-1}$ ) (Rodrigues \& Almeida, 1998; Vieira \& Silva, 1998). O solo empregado neste estudo continha na camada de solo de $0-15 \mathrm{~cm}$ mais de 2 dag kg-1 de matéria orgânica, além de 49 e $43 \mathrm{dag} \mathrm{kg}^{-1}$ de argila no plantio direto e no convencional, respectivamente. Isso explica a presença do herbicida apenas na camada de solo de $0-5 \mathrm{~cm}$, no plantio convencional, além da ausência de resíduos vegetais nesse sistema de plantio. Em estudo conduzido por Wiese \& Turner (1977), em solo com 1,5 dag $\mathrm{kg}^{-1}$ de matéria orgânica e 33 dag $\mathrm{kg}^{-1}$ de argila, o metolachlor foi deslocado até $10 \mathrm{~cm}$ de profundidade quando aplicado com lâminas de água de $33 \mathrm{~mm}$, porém ele não se movimentou além de $5 \mathrm{~cm}$ com $20 \mathrm{~mm}$ de

Tabela 2 - Massa seca de plantas de sorgo cultivadas em amostras de solo coletadas em diferentes profundidades, 15 dias após a herbigação ou a pulverização do metolachlor $\left(2,4 \mathrm{~kg} \mathrm{ha}^{-1}\right)$. Coimbra-MG, 1999

\begin{tabular}{|c|c|c|c|c|c|c|c|}
\hline \multirow{3}{*}{\multicolumn{2}{|c|}{ Tratamento }} & \multicolumn{6}{|c|}{ Porcentagem da Massa Seca de Plantas de Sorgo ${ }^{1 /}$} \\
\hline & & \multicolumn{3}{|c|}{ Plantio Direto } & \multicolumn{3}{|c|}{ Convencional } \\
\hline & & $0-5 \mathrm{~cm}$ & $5-10 \mathrm{~cm}$ & $10-15 \mathrm{~cm}$ & $0-5 \mathrm{~cm}$ & $5-10 \mathrm{~cm}$ & $10-15 \mathrm{~cm}$ \\
\hline \multirow{3}{*}{ Herbigação } & $5 \mathrm{~mm}$ & $99 \pm 1,1$ & $99 \pm 1,7$ & $98 \pm 1,7$ & $65 \pm 5,1$ & 100 & $94 \pm 1,1$ \\
\hline & $10 \mathrm{~mm}$ & $97 \pm 2,5$ & $97 \pm 4,0$ & $98 \pm 2,3$ & $70 \pm 3,4$ & $96 \pm 1,1$ & $96 \pm 1,7$ \\
\hline & $15 \mathrm{~mm}$ & $97 \pm 2,6$ & $96 \pm 0,5$ & $99 \pm 1,1$ & $69 \pm 3,0$ & $98 \pm 2,1$ & $98 \pm 2,5$ \\
\hline \multicolumn{2}{|c|}{ Pulverização $\left(200 \mathrm{~L} \mathrm{ha}^{-1}\right)$} & $98 \pm 2,8$ & $94 \pm 3,0$ & 100 & $97 \pm 2,1$ & $99 \pm 1,1$ & $97 \pm 2,8$ \\
\hline \multicolumn{2}{|c|}{ Testemunha } & 100 & 100 & 100 & 100 & 100 & 100 \\
\hline
\end{tabular}

${ }^{1 /}$ Em relação à testemunha.

Tabela 3 - Massa seca de plantas de sorgo cultivadas em amostras de solo coletadas em diferentes profundidades, 30 dias após a herbigação ou a pulverização do metolachlor $\left(2,4 \mathrm{~kg} \mathrm{ha}^{-1}\right)$. Coimbra-MG, 1999

\begin{tabular}{|c|c|c|c|c|c|c|c|}
\hline \multirow{3}{*}{\multicolumn{2}{|c|}{ Tratamento }} & \multicolumn{6}{|c|}{ Porcentagem da Massa Seca de Plantas de Sorgo ${ }^{1 /}$} \\
\hline & & \multicolumn{3}{|c|}{ Plantio Direto } & \multicolumn{3}{|c|}{ Convencional } \\
\hline & & $0-5 \mathrm{~cm}$ & $5-10 \mathrm{~cm}$ & $10-15 \mathrm{~cm}$ & $0-5 \mathrm{~cm}$ & $5-10 \mathrm{~cm}$ & $10-15 \mathrm{~cm}$ \\
\hline \multirow{3}{*}{ Herbigação } & $5 \mathrm{~mm}$ & $94+2,3$ & $96+2,4$ & $96+2,6$ & $94+4,7$ & $96+4,0$ & $98+2,8$ \\
\hline & $10 \mathrm{~mm}$ & $94 \pm 3,2$ & $97 \pm 3,0$ & $99 \pm 1,0$ & $96 \pm 3,2$ & $98 \pm 3,4$ & $96 \pm 4,0$ \\
\hline & $15 \mathrm{~mm}$ & $94 \pm 4,7$ & $98 \pm 2,0$ & $97 \pm 2,0$ & $96 \pm 3,6$ & $97 \pm 2,0$ & $95 \pm 1,5$ \\
\hline \multicolumn{2}{|c|}{ Pulverização $\left(200 \mathrm{~L} \mathrm{ha}^{-1}\right)$} & $97 \pm 3,7$ & $99 \pm 0,5$ & $97 \pm 1,1$ & $96 \pm 3,7$ & $98 \pm 3,4$ & $95 \pm 4,5$ \\
\hline \multicolumn{2}{|c|}{ Testemunha } & 100 & 100 & 100 & 100 & 100 & 100 \\
\hline
\end{tabular}

${ }^{1 /}$ Em relação à testemunha.

Tabela 4 - Massa seca de plantas de sorgo cultivadas em amostras de solo coletadas em diferentes profundidades, 45 dias após a herbigação ou a pulverização do metolachlor $\left(2,4 \mathrm{~kg} \mathrm{ha}^{-1}\right)$. Coimbra-MG, 1999

\begin{tabular}{|c|c|c|c|c|c|c|c|}
\hline \multirow{3}{*}{\multicolumn{2}{|c|}{ Tratamento }} & \multicolumn{6}{|c|}{ Porcentagem da Massa Seca de Plantas de Sorgo $0^{1 /}$} \\
\hline & & \multicolumn{3}{|c|}{ Plantio Direto } & \multicolumn{3}{|c|}{ Convencional } \\
\hline & & $0-5 \mathrm{~cm}$ & $5-10 \mathrm{~cm}$ & $10-15 \mathrm{~cm}$ & $0-5 \mathrm{~cm}$ & $5-10 \mathrm{~cm}$ & $10-15 \mathrm{~cm}$ \\
\hline \multirow{3}{*}{ Herbigação } & $5 \mathrm{~mm}$ & $96 \pm 2,0$ & $96 \pm 4,0$ & $98 \pm 1,7$ & $93 \pm 2,3$ & $92 \pm 8,4$ & $93 \pm 7,2$ \\
\hline & $10 \mathrm{~mm}$ & $96 \pm 3,4$ & $95 \pm 2,0$ & $95 \pm 6,0$ & $95 \pm 4,8$ & $96 \pm 3,4$ & $95 \pm 7,2$ \\
\hline & $15 \mathrm{~mm}$ & $96 \pm 6,2$ & $97 \pm 5,1$ & $97 \pm 5,2$ & $93 \pm 4,3$ & $92 \pm 4,8$ & $95 \pm 5,4$ \\
\hline \multicolumn{2}{|c|}{ Pulverização $\left(200 \mathrm{~L} \mathrm{ha}^{-1}\right)$} & $99 \pm 1,7$ & $96 \pm 4,5$ & $94 \pm 6,5$ & $95 \pm 5,5$ & $92 \pm 2,0$ & $93 \pm 5,6$ \\
\hline \multicolumn{2}{|c|}{ Testemunha } & 100 & 100 & 100 & 100 & 100 & 100 \\
\hline
\end{tabular}

1/ Em relação à testemunha. 
Tabela 5 - Massa seca de plantas de sorgo cultivadas em amostras de solo coletadas em diferentes profundidades, 60 dias após a herbigação ou a pulverização do metolachlor $\left(2,4 \mathrm{~kg} \mathrm{ha}^{-1}\right)$. Coimbra-MG, 1999

\begin{tabular}{|c|c|c|c|c|c|c|c|}
\hline \multirow{3}{*}{\multicolumn{2}{|c|}{ Tratamento }} & \multicolumn{6}{|c|}{ Porcentagem da Massa Seca de Plantas de Sorgo ${ }^{1 /}$} \\
\hline & & \multicolumn{3}{|c|}{ Plantio Direto } & \multicolumn{3}{|c|}{ Convencional } \\
\hline & & $0-5 \mathrm{~cm}$ & $5-10 \mathrm{~cm}$ & $10-15 \mathrm{~cm}$ & $0-5 \mathrm{~cm}$ & $5-10 \mathrm{~cm}$ & $10-15 \mathrm{~cm}$ \\
\hline \multirow{3}{*}{ Herbigação } & $5 \mathrm{~mm}$ & $94 \pm 3,6$ & $94 \pm 4,7$ & $98 \pm 2,8$ & $95 \pm 4,5$ & $92 \pm 3,0$ & $96 \pm 3,5$ \\
\hline & $10 \mathrm{~mm}$ & $94 \pm 3,2$ & $96 \pm 8,9$ & $93 \pm 7,5$ & $95 \pm 2,8$ & $95 \pm 4,5$ & $97 \pm 4,0$ \\
\hline & $15 \mathrm{~mm}$ & $95 \pm 4,5$ & $97 \pm 5,1$ & $95 \pm 4,5$ & $95 \pm 3,2$ & $96 \pm 3,5$ & $92 \pm 6,0$ \\
\hline \multicolumn{2}{|c|}{ Pulverização $\left(200 \mathrm{~L} \mathrm{ha}^{-1}\right)$} & $92 \pm 5,1$ & $96 \pm 5,5$ & $94 \pm 3,6$ & $99 \pm 0,5$ & $95 \pm 4,5$ & $96 \pm 0,5$ \\
\hline \multicolumn{2}{|c|}{ Testemunha } & 100 & 100 & 100 & 100 & 100 & 100 \\
\hline
\end{tabular}

Em relação à testemunha.

água. Em solo franco-siltoso $\left(0,94 \mathrm{dag} \mathrm{kg}^{-1} \mathrm{de}\right.$ matéria orgânica e 7,3 dag $\mathrm{kg}^{-1}$ de argila), o metolachlor aplicado com lâmina de água de $13 \mathrm{~mm}$ atingiu $30 \mathrm{~cm}$ de profundidade (Barnes et al., 1992).

No plantio direto, diferentemente do plantio convencional, o herbicida geralmente não foi detectado 15 dias após a sua aplicação em nenhuma das camadas de solo (Tabela 2). Uma das explicações para esse fato foi a provável retenção de parte do metolachlor na palhada que havia sobre o solo. Os resíduos vegetais têm grande capacidade de sorção, às vezes maior que a do solo (Dao, 1995; Reddy et al., 1995). Conseqüentemente, nessas condições, o produto ficou mais sujeito a perdas por fotodecomposição e/ou volatilização. Além disso, é possível que o metolachlor que atingiu o solo em plantio direto tenha sido mais rapidamente degradado que no solo do plantio convencional. A razão disso é o maior conteúdo relativo de matéria orgânica na superficie do solo em plantio direto $\left(2,83 \mathrm{dag} \mathrm{kg}^{-1}\right.$ contra 2,57 dag $\mathrm{kg}^{-1}$ no plantio convencional). De acordo com Braverman et al. (1986), a atividade microbiana está diretamente relacionada com o conteúdo de matéria orgânica do solo, e ela é o principal fator que governa a persistência do metolachlor no solo.

Observa-se, nas Tabelas 6, 7, 8 e 9, que o método de aplicação e o sistema de plantio não influenciaram a lixiviação do fomesafen no solo, que se concentrou na camada de $0-10 \mathrm{~cm}$ de profundidade.

Resultado semelhante foi obtido por Cobucci (1996) em área com preparo convencional. Esse autor verificou que grande parte do fomesafen aplicado na cultura do feijão, nas doses de 0,125;0,25;0,375 e $0,5 \mathrm{~kg} \mathrm{ha}^{-1}$, em um Latossolo Vermelho-Escuro com 71 dag $\mathrm{kg}^{-1}$ de argila e 3,92 dag $\mathrm{kg}^{-1}$ de matéria orgânica, concentrou-se na camada de solo de $0-10 \mathrm{~cm}$ de profundidade. Naquele estudo, as parcelas receberam um total de água (chuva e irrigação) de, aproximadamente, $680 \mathrm{~mm}$, no período de condução do experimento (212 dias); apenas pequena parte do herbicida deslocou-se além da camada de 0-10 cm. Segundo Weber et al. (1993) e Cobucci (1996), a baixa mobilidade do fomesafen nos solos brasileiros pode ser atribuída à sua sorção aos colóides orgânicos e minerais do solo e aos óxidos de ferro e aluminio, apesar de sua elevadíssima solubilidade em água (600.000 $\mu \mathrm{g} \mathrm{L}^{-1}$, formulação salina de sódio) e $\mathrm{K}_{\mathrm{oc}}$ considerado baixo (60 $\mathrm{mL} \mathrm{g}^{-1}$ de solo) (Rodrigues \& Almeida, 1998).

Esperava-se que o fomesafen se movimentasse a maiores profundidades que no solo testado por Cobucci (1996), em razão dos menores conteúdos de argila e de matéria orgânica nas áreas de plantio direto (49 e 2,83 dag $\mathrm{kg}^{-1}$ de argila e matéria orgânica, respectivamente) e convencional (43 e 2,57 dag kg-1 de argila e matéria orgânica, respectivamente). Possivelmente, o fomesafen não tenha se deslocado além da camada de $0-10 \mathrm{~cm}$ devido ao menor volume de água (chuva e irrigação) recebido na lavoura, que totalizou $264 \mathrm{~mm}$. Esse volume de água foi aproximadamente três vezes menor que o verificado no trabalho conduzido por Cobucci (1996), que foi de $680 \mathrm{~mm}$. Outro fator que pode ter influenciado o menor deslocamento do fomesafen foi o tempo de condução do experimento (60 dias após a aplicação do herbicida). 
Tabela 6 - Massa seca de plantas de sorgo cultivadas em amostras de solo coletadas em diferentes profundidades, 15 dias após a herbigação e a pulverização do fomesafen $\left(0,225 \mathrm{~kg} \mathrm{ha}^{-1}\right)$. Coimbra-MG, 1999

\begin{tabular}{|c|c|c|c|c|c|c|c|}
\hline \multirow{3}{*}{\multicolumn{2}{|c|}{ Tratamento }} & \multicolumn{6}{|c|}{ Porcentagem da Massa Seca de Plantas de Sorgo ${ }^{1 /-}$} \\
\hline & & \multicolumn{3}{|c|}{ Plantio Direto } & \multicolumn{3}{|c|}{ Convencional } \\
\hline & & $0-5 \mathrm{~cm}$ & $5-10 \mathrm{~cm}$ & $10-15 \mathrm{~cm}$ & $0-5 \mathrm{~cm}$ & $5-10 \mathrm{~cm}$ & $10-15 \mathrm{~cm}$ \\
\hline \multirow{3}{*}{ Herbigação } & $3 \mathrm{~mm}$ & $30+10,5$ & $63 \pm 9,2$ & $94 \pm 6,8$ & $28+9,5$ & $61 \pm 8,2$ & $95 \pm 3,3$ \\
\hline & $6 \mathrm{~mm}$ & $36 \pm 9,7$ & $60 \pm 9,5$ & $92 \pm 2,0$ & $29 \pm 2,1$ & $63 \pm 5,7$ & $95 \pm 4,5$ \\
\hline & $9 \mathrm{~mm}$ & $30 \pm 13,6$ & $65 \pm 9,7$ & $95 \pm 2,0$ & $32 \pm 8,7$ & $62 \pm 9,5$ & $93 \pm 7,1$ \\
\hline \multicolumn{2}{|c|}{ Pulverização $\left(200 \mathrm{~L} \mathrm{ha}^{-1}\right)$} & $33 \pm 6,0$ & $66 \pm 4,0$ & $95 \pm 2,6$ & $33 \pm 9,9$ & $58 \pm 4,1$ & $94 \pm 4,7$ \\
\hline \multicolumn{2}{|c|}{ Testemunha } & 100 & 100 & 100 & 100 & 100 & 100 \\
\hline
\end{tabular}

1/ Em relação à testemunha.

Tabela 7 - Massa seca de plantas de sorgo cultivadas em amostras de solo coletadas em diferentes profundidades, 30 dias após a herbigação e a pulverização do fomesafen $\left(0,225 \mathrm{~kg} \mathrm{ha}^{-1}\right)$. Coimbra-MG, 1999

\begin{tabular}{|c|c|c|c|c|c|c|c|}
\hline \multirow{3}{*}{\multicolumn{2}{|c|}{ Tratamento }} & \multicolumn{6}{|c|}{ Porcentagem da Massa Seca de Plantas de Sorgo ${ }^{1 /}$} \\
\hline & & \multicolumn{3}{|c|}{ Plantio Direto } & \multicolumn{3}{|c|}{ Convencional } \\
\hline & & $0-5 \mathrm{~cm}$ & $5-10 \mathrm{~cm}$ & $10-15 \mathrm{~cm}$ & $0-5 \mathrm{~cm}$ & $5-10 \mathrm{~cm}$ & $10-15 \mathrm{~cm}$ \\
\hline \multirow{3}{*}{ Herbigação } & $3 \mathrm{~mm}$ & $34+5,5$ & $60 \pm 4,0$ & $95 \pm 4,1$ & $34+4,1$ & $65 \pm 4,5$ & $96+3,5$ \\
\hline & $6 \mathrm{~mm}$ & $37 \pm 6,8$ & $62 \pm 8,5$ & $97 \pm 2,1$ & $36 \pm 9,0$ & $61 \pm 5,5$ & $96 \pm 4,0$ \\
\hline & $9 \mathrm{~mm}$ & $34 \pm 7,5$ & $63+9,1$ & $97 \pm 4,6$ & $40 \pm 5,5$ & $62 \pm 7,6$ & $94+3,8$ \\
\hline \multicolumn{2}{|c|}{ Pulverização $\left(200 \mathrm{~L} \mathrm{ha}^{-1}\right)$} & $36 \pm 5,7$ & $65 \pm 8,0$ & $92 \pm 4,7$ & $38 \pm 3,5$ & $61 \pm 9,6$ & $98 \pm 3,4$ \\
\hline \multicolumn{2}{|c|}{ Testemunha } & 100 & 100 & 100 & 100 & 100 & 100 \\
\hline
\end{tabular}

1/ Em relação à testemunha.

Tabela 8 - Massa seca de plantas de sorgo cultivadas em amostras de solo coletadas em diferentes profundidades, 45 dias após a herbigação e a pulverização do fomesafen $\left(0,225 \mathrm{~kg} \mathrm{ha}^{-1}\right)$. Coimbra-MG, 1999

\begin{tabular}{|c|c|c|c|c|c|c|c|}
\hline \multirow{2}{*}{\multicolumn{2}{|c|}{ Tratamento }} & \multicolumn{6}{|c|}{ Porcentagem da Massa Seca de Plantas de Sorgo } \\
\hline & & \multicolumn{3}{|c|}{ Plantio Direto } & \multicolumn{3}{|c|}{ Convencional } \\
\hline & & $0-5 \mathrm{~cm}$ & $5-10 \mathrm{~cm}$ & $10-15 \mathrm{~cm}$ & $0-5 \mathrm{~cm}$ & $5-10 \mathrm{~cm}$ & $10-15 \mathrm{~cm}$ \\
\hline \multirow{3}{*}{ Herbigação } & $3 \mathrm{~mm}$ & $33 \pm 6,4$ & $64 \pm 6,2$ & $94 \pm 5,1$ & $36 \pm 7,1$ & $56 \pm 7,1$ & $93 \pm 5,0$ \\
\hline & $6 \mathrm{~mm}$ & $36 \pm 4,9$ & $61 \pm 4,1$ & $95 \pm 2,6$ & $40 \pm 5,2$ & $56 \pm 9,9$ & $96 \pm 4,7$ \\
\hline & $9 \mathrm{~mm}$ & $38 \pm 9,7$ & $65 \pm 9,3$ & $93 \pm 4,7$ & $32 \pm 8,1$ & $64 \pm 10,0$ & $97 \pm 4,0$ \\
\hline \multicolumn{2}{|c|}{ Pulverizacão $\left(200 \mathrm{~L} \mathrm{ha}^{-1}\right)$} & $38 \pm 7,5$ & $65 \pm 8,3$ & $98 \pm 2,8$ & $42 \pm 3,0$ & $68 \pm 9,1$ & $96 \pm 1,0$ \\
\hline \multicolumn{2}{|c|}{ Testemunha } & 100 & 100 & 100 & 100 & 100 & 100 \\
\hline
\end{tabular}

${ }^{1 /}$ Em relação à testemunha.

Tabela 9 - Massa seca de plantas de sorgo cultivadas em amostras de solo coletadas em diferentes profundidades, 60 dias após a herbigação e a pulverização do fomesafen $\left(0,225 \mathrm{~kg} \mathrm{ha}^{-1}\right)$. Coimbra-MG, 1999

\begin{tabular}{|c|c|c|c|c|c|c|c|}
\hline \multirow{3}{*}{\multicolumn{2}{|c|}{ Tratamento }} & \multicolumn{6}{|c|}{ Porcentagem da Massa Seca de Plantas de Sorgo ${ }^{-1}$} \\
\hline & & \multicolumn{3}{|c|}{ Plantio Direto } & \multicolumn{3}{|c|}{ Convencional } \\
\hline & & $0-5 \mathrm{~cm}$ & $5-10 \mathrm{~cm}$ & $10-15 \mathrm{~cm}$ & $0-5 \mathrm{~cm}$ & $5-10 \mathrm{~cm}$ & $10-15 \mathrm{~cm}$ \\
\hline \multirow{3}{*}{ Herbigação } & $3 \mathrm{~mm}$ & $35 \pm 9,5$ & $64 \pm 9,1$ & $96 \pm 2,8$ & $28 \pm 4,0$ & $52 \pm 7,0$ & $93 \pm 3,4$ \\
\hline & $6 \mathrm{~mm}$ & $33 \pm 9,1$ & $61 \pm 1,5$ & $94 \pm 4,1$ & $31 \pm 6,6$ & $58+14,0$ & $92 \pm 7,5$ \\
\hline & $9 \mathrm{~mm}$ & $32 \pm 5,5$ & $58 \pm 8,9$ & $95 \pm 4,3$ & $24 \pm 6,6$ & $57 \pm 9,4$ & $93 \pm 3,5$ \\
\hline \multicolumn{2}{|c|}{ Pulverização (200 L ha" $\left.{ }^{-1}\right)$} & $35 \pm 4,1$ & $58 \pm 3,1$ & $95 \pm 2,6$ & $34 \pm 5,2$ & $63 \pm 4,5$ & $95 \pm 3,8$ \\
\hline \multicolumn{2}{|c|}{ Testemunha } & 100 & 100 & 100 & 100 & 100 & 100 \\
\hline
\end{tabular}

1/ Em relação à testemunha. 
Os resultados obtidos também mostraram que a persistência no solo do fomesafen foi, provavelmente, além de 60 dias. Segundo Rodrigues \& Almeida (1998), a sua persistência no solo varia de 60 a 180 dias.

A movimentação no solo dos herbicidas metolachlor e fomesafen aplicados com diferentes lâminas de água foi ambientalmente segura, pouco diferindo em relação à pulverização. Portanto, em ambos os sistemas de plantio a herbigação pode ser realizada com lâminas de água relativamente altas.

\section{LITERATURA CITADA}

AHRENS, W. H. Herbicide handbook. 7.ed. Lawrence: Weed Science Society of America, 1994. p. 352.

ASSOCIAÇÃO BRASILEIRA DE NORMAS TÉCNICAS ABNT. Sistema de irrigação por aspersão pivô central: caracterização de desempenho/método de ensaio. Rio de Janeiro. 1985. 22 p. (Projeto de norma, 12:02.08-005).

BARNES, C. J.; LAVY, T. L.; TALBERT, R. E. Leaching, dissipation, and efficacy of metolachlor applied by chemigation or conventional methods. J. Environ. Quality., v. 21, n. 2, p. 232-236, 1992.

BRAVERMAN, M. P.; LAVY, T. L.; BARNES, C. J. Degradation and bioactivity of metolachlor in the soil. Weed Sci., v. 34, n. 3, p. 479-484, 1986.

BURGARD, D. J. et al. Metolachlor distribution in a sandy soil under irrigated potato production. Weed Sci., v. 41, n. 4, p.648-655, 1993.

COBUCCI, T. Avaliação agronômica dos herbicidas fomesafen e bentazon e efeito de seus resíduos no ambiente, no sistema irrigado feijão-milho. 1996. 106 f. Tese (Doutorado em Fitotecnia) - Universidade Federal de Viçosa, Viçosa, 1996.

DAO, T. H. Field decay of wheat straw and its effects on metribuzin and s-ethyl metribuzin sorption and elution from crop residues. J. Environ. Quality, v. 20, n. 1, p.203-208, 1991.

DAO, T. H. Subsurface mobility of metribuzin as affected by crop residue placement and tillage method. J. Environ. Quality, v. 24, n. 6, p. 1193-1198, 1995.

GUSTAFSON, D. I. Groundwater ubiquity score: a simple method for assessing pesticide leachibility. Environ.

Toxicol. Chem., v. 8, n. 4, p. 339-357, 1989.

Planta Daninha, Viçosa-MG, v. 22, n. 4, p. 623-631, 2004
HALL, J. K.; MURRAY, M. R.; HARTWIG, N. L. Herbicide leaching and distribution in tilled and untilled soil. J. Environ. Quality, v. 18, n. 4, p. 439-445, 1989.

HEATWOLE, C. D. et al. Movement of field-applied atrazine, metolachlor, and bromide in a sandy loam soil. Trans. Am. Soc. Agron. Eng., v. 40, n. 5, p. 1267-1276, 1997.

INOUE, M. H. et al. Critérios para avaliação do potencial de lixiviação dos herbicidas comercializados no estado do Paraná. Planta Daninha, v. 21, n. 2, p. 313-323, 2003.

ISENSEE, A. R.; NASH, R. G.; HELLING, C. S. Effect of conventional vs. no-tillage on pesticide leaching to shallow groundwater. J. Environ. Quality, v. 19, n. 3, p. 434-440, 1990.

ISENSEE, A. R.; SADEGHI, A. M. Long-term effect of tillage and rainfall on herbicide leaching to shallow groundwater. Chemosphere, v. 30, n. 4, p. 671-685, 1995.

KELLER, K. E.; WEBER, J. B. Soybean (Glycine max) influences metolachlor mobility in soil. Weed Sci., v. 45, n. 6 , p. 833-841, 1997.

KELLER, K. E. et al. Temporal distribution of ${ }^{14} \mathrm{C}$ in soil water from field lysimeters treated with ${ }^{14} \mathrm{C}$-metolachlor. Soil Sci., v. 163, n. 11, p. 872-882, 1998.

LEE, R. F.; WEBER, J. B. Influence of polymers on the mobility, loss, and bioactivity of ${ }^{14} \mathrm{C}$ from ${ }^{14} \mathrm{C}$-labeled atrazine, metolachlor, and primisulfuron. J. Agr. Food. Chem., v. 41, n. 6, p. 988-995, 1993.

MANTOVANI, E. C.; RAMOS, M. M. Manejo da irrigação. In: COSTA, E. F.; VIEIRA, R. F.; VIANA, P. A. Quimigação - aplicação de produtos químicos e biológicos via irrigação. Brasília: Embrapa/SPI, 1994. p. 129-158.

OLIVEIRA JR., R. S. Relação entre propriedades químicas e físicas do solo e sorção, dessorção e potencial de lixiviação de herbicidas. 1998. 83 f. Tese (Doutorado em Fitotecnia) - Universidade Federal de Viçosa, Viçosa, 1998.

PROCÓPIO, S. O. et al. Efeito da irrigação inicial na profundidade de lixiviação do herbicida S-metolachlor em diferentes tipos de solos. Planta Daninha, v. 19, n. 3, p. 409-417, 2001.

REDDY, K. N. et al. Chlorimuron ethyl sorption and desorption kinetics in soils and herbicide-desiccated cover crop 3. J. Agr. Fd. Chem., v. 43, n. 10, p. 2752-2757, 1995. 
RITTER, W. F.; SCARBOROUGH, R. W.; CHIRNSIDE, A. E. M. Herbicide leaching in coastal plain soil. J. Irrig. Drain. Engin., v. 120, n. 3, p. 634-649, 1994.

RODRIGUES, B. N.; ALMEIDA, F. R. Guia de herbicidas. 4.ed. Londrina: Edição dos Autores, 1998. p. 648.

SIGUA, G. C.; ISENSEE, A. R.; SADEGHI, A. M. Influence of rainfall intensity and crop residue on leaching of atrazine through intact no-till soil cores. Soil Sci., v. 156, n. 4, p. 225-232, 1993.

STARR, J. L.; GLOTFELTY, D. E. Atrazine and bromide movement trough a silt loam soil. J. Environ. Quality, v. 19, n. 3, p. 552-558, 1990.

THREADGILL, E. D. Current status and future of chemigation. In: NATIONAL SYMPOSIUM ON CHEMIGATION, 3., 1985, Tifton. Proceedings... Tifton: Rural Development Center, 1985. p. 1-12.
VIEIRA, R. F.; SILVA, A. A. Aplicação de defensivos via água de irrigação por aspersão. In: VIEIRA, C.; PAULA JR., T. J.; BORÉM, A. Feijão - aspectos gerais e cultura no Estado de Minas. Viçosa: UFV, 1998. p. 267-323.

WEBER, J. B. Ionization and sorption of fomesafen and atrazine by soils and soil constituents. Pestic. Sci., v. 39, n. 1, p. 31-38, 1993.

WEBER, J. B.; STREK, H. J.; SARTORI, J. L. Mobility of fomesafen and atrazine in soil columns under satured and unsatured flow conditions. Pestic. Sci., v. 39, n. 1, p. 3946,1993 .

WIESE, A. F.; TURNER, W. E. Herbicide application with sprinkler irrigation. Southern Weed Science Society, n. 31, p. 100, 1977.

WILSON, G. V. et al. Tillage and cover crop effects on saturated and unsaturated transport of fluometuron. Soil Sci. Soc. Am. J., v. 62, n. 1, p. 46-55, 1998. 